\title{
Разработка макета волоконно-оптического бесконтактного разъема с помощью технологии Expanded Beam
}

\author{
Я. Д. Токарева ${ }^{1}$, А. И. Гаранин ${ }^{2,3}$, А. Ю. Петухова ${ }^{2,3}$, Ю. А. Конин ${ }^{3}$, \\ В. А. Щербакова ${ }^{3,4}$ \\ ${ }^{1}$ Университет информационных технологий, механики и оптики \\ 196135, Санкт-Петербург, ул. Кронверский проспект, 49 \\ 2 Пермский национальный исследовательский политехнический университет \\ 614990, Пермь, ул. Комсомольский проспект, 79 \\ 3 ПАО «Пермская научно-производственная приборостроительная компания» \\ 614990, Пермь, ул. 25 Октября, 106 \\ ${ }^{4}$ Пермский государственный национальный исследовательский университет \\ 614990, Пермь, ул. Букирева, 15
}

\begin{abstract}
Рассматривается макет бесконтактного волоконно-оптического разъема для систем доставки мощного лазерного излучения в полевых условиях эксплуатации с помощью технологии Expanded Beam («расширитель пучка»). Реализуемая технология, в отличие от контактной, способствует уменьшению оптических потерь от загрязнения и смещения сердцевин оптических волокон. Диаметр пучка после прохождения линзы становится в 15 раз больше, чем сердцевина оптического волокна, тем самым уменьшая потери от частичек пыли на торцах оптических волокон, которые из-за своих размеров могут вносить значительные потери. В таких разъемах предлагается использовать шариковые линзы, поскольку их удобно устанавливать, и они относительно не дороги. Сам разъем представляет собой конструкцию с шариковыми линзами, излучение на которых сначала преобразуется в параллельный световой пучок большего диаметра, а затем снова фокусируется на торец выходного волокна. Была разработана модель, позволяющая рассчитывать такое расположение сферических линз относительно друг друга и относительно оптических волокон, при котором потери будут минимальными. Макет разъема был собран из следующих компонентов: многомодовое оптическое волокно доставки, лазерный диод накачки 975 нм, микроподвижки, шариковые линзы и измеритель оптической мощности. В качестве материалов линз использовались стекло марки $\mathrm{K}-8$ и прозрачный оксид алюминия $\mathrm{Al}_{2} \mathrm{O}_{3}$, традиционно называемый в оптике сапфиром. Была исследована зависимость между коэффициентом пропускания излучения и диаметром шариковых линз. Такой разъем может применяться в системах доставки лазерного излучения, таких как разрывные пиропатроны в аэрокосмической и горнодобывающей промышленности.
\end{abstract}

Ключевые слова: Expanded Beam; расширенный пучок; оптическое волокно

\section{Layout design fiber optic proximity connector with Expanded Beam technology}




\section{Ya. D. Tokareva ${ }^{1}$, A. I. Garanin ${ }^{2,3}$, A. Yu. Petukhova ${ }^{2,3}$, Yu. A. Konin ${ }^{3}$,} V. A. Scherbackova $\mathbf{a}^{3,4}$

${ }^{1}$ ITMO University, Kronverskiy prospect St. 49, 196135, St. Petersburg

${ }^{2}$ Perm National Research Polytechnic University, Komsomolskiy prospect St. 79, 614990, Perm

${ }^{3}$ Perm research and production instrument-making company "PNPPK", 25 Oktyabrya st. 106, Perm

${ }^{4}$ Perm State University, Bukireva St. 15, 614990, Perm

This article discusses the layout of a non-contact fiber optic connector for high-power laser radiation delivery systems in the field using Expanded Beam technology (Beam Expander). The technology being implemented, in contrast to contact technology, helps to reduce optical loss from contamination and displacement of the core of optical fibers. The diameter of the beam after passing through the lens becomes 15 times larger than the core of the optical fiber, thereby reducing losses from dust particles at the ends of the optical fibers, which, due to their size, can introduce significant losses. In such connectors, it is proposed to use ball lenses since they are convenient to install and relatively inexpensive. The connector itself is a design with ball lenses, the radiation on which is first converted into a parallel light beam of a larger diameter, and then focuses again on the end of the output fiber. A model was developed, thanks to which the locations of spherical lenses were calculated relative to each other and relative to optical fibers, at which losses would be minimal. The prototype of the connector was assembled from the following components: multimode optical fiber delivery, laser pump diode $975 \mathrm{~nm}$, micromotors, ball lenses and optical power meter. K-8 glass and transparent aluminum oxide $\mathrm{Al} 2 \mathrm{O} 3$, traditionally called sapphire in optics, were used as lens materials. The relationship between the transmittance of radiation and the diameter of ball lenses was investigated. Such a connector can be used in laser delivery systems, such as explosive squibs in the aerospace and mining industries.

Keywords: Expanded Beam; optical fiber; spatial resolution

Received 13.08.2019; accepted 03.02.2020

doi: 10.17072/1994-3598-2020-1-05-10

\section{1. Введение}

В основе большинства современных волоконно-оптических коннекторов лежит принцип физического контакта (PC - Physical Contact), который представляет собой механический контакт двух наконечников (ferrule), устанавливаемых на концах соединяемых оптических волокон. При условии качественной полировки торцов наконечников и высокой точности их взаимного расположения такие соединения характеризуются низкой величиной вносимых потерь $(<1 \mathrm{~dB})$.

Оптические разъемы, основанные на принципе физического контакта, чувствительны к изменениям внешней среды и требуют соблюдения достаточно жестких правил эксплуатации [1]. Это ограничивает возможность применения таких соединений в полевых условиях и при воздействии агрессивных сред.

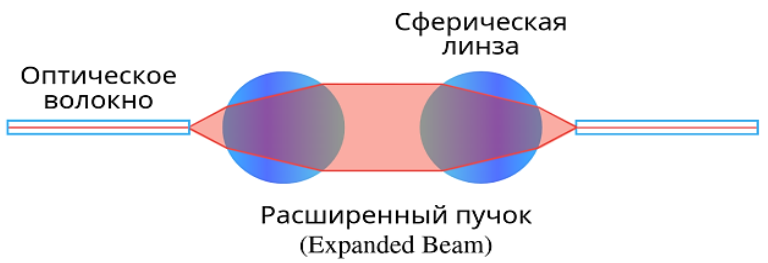

Рис. 1. Схема технологии Expanded Beam

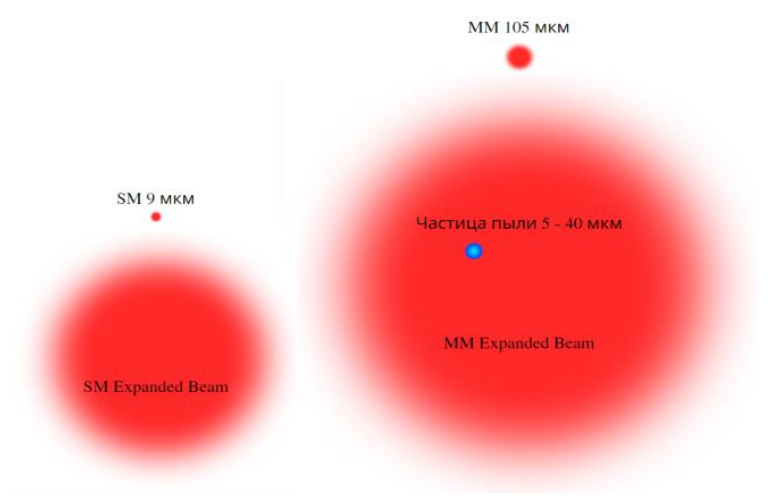

Рис. 2. Сравнение профиля сечения расииренного пучка и диаметров сердиевин оптических волокон с инородными частицุами

Увеличение диаметра пучка в сотни и даже тысячи раз по сравнению с диаметром сердцевины волокна (рис. 2) сводит к минимуму влияние инородных частиц, которые могут попасть на поверхность разъема. Отсутствие прямого контакта между линзами способствует уменьшению износа разъема и, как следствие, увеличению срока службы.

Для решения этой проблемы авторами статьи была разработана и исследована конструкция, со- 
стоящая из двух шариковых линз. В основе этого соединения лежит бесконтактная технология соединения излучения оптических волокон, получившая название Expanded Beam («расширенный пучок») [2-4]. Среди ее преимуществ можно выделить меньшую проницаемость для загрязнений и повреждений [5-9]. В этом случае на торцах соединяемых волокон устанавливаются сферические линзы, с помощью которых свет, распространяющийся в волокне, сначала преобразуется в параллельный световой пучок большего диаметра, а затем снова фокусируется на торец второго волокна. Условное изображение принципа работы технологии Expanded Beam приведено на рис. 1.

На сегодня данная тема является актуальной, поскольку разъемы Expanded Beam используются для высокомощных лазеров, телекоммуникационных линий передач при мощностях от 1-5 вт и медицинских лазеров, где есть возможность перекручивания волокна [10].

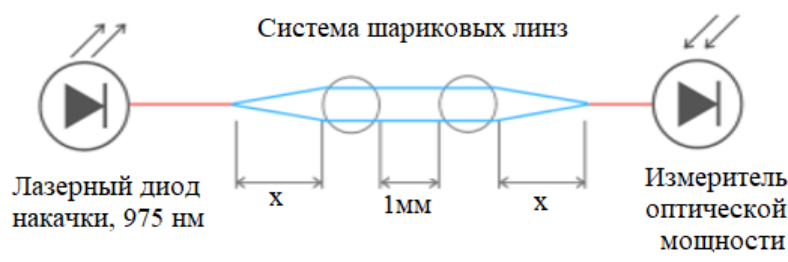

Рис. 3. Принциипиальная схема установки

Цель данной работы заключалась в исследовании оптических потерь для оптической системы линз разных диаметров и показателей преломления. Для доставки излучения использовались многомодовые оптические волокна кварц-кварц (Nufern 105/125). Для будущего изготовления расчеты проводились для симметричной системы, т.е. зазор между торцом оптического волокна и линзы был одинаковый как для первой, так и для второй шариковой линзы (рис. 3).

\section{2. Теоретический расчет}

В данной работе, при соблюдении условия компактности коннектора были использованы шариковые линзы диаметрами 1, 2 и 3 мм. В качестве материалов линз - стекла марки $\mathrm{K}-8$ и $\mathrm{Al}_{2} \mathrm{O}_{3}$ (сапфир).

Для расчета условия коллимации для шариковых линз применялись уравнения (1), (2) и (3). Для полного понимания картины расчета все параметры линзы были изображены на рис. 4.

- Эффективное фокусное расстояние шариковой линзы (EFL) рассчитывается как

$$
E F L=\frac{n D}{4(n-1)} .
$$

- Заднее фокусное расстояние шарового объектива (BFL) вычисляется как

$$
B F L=E F L-\frac{D}{2}
$$

- Диаметр источника выходного сигнала находится как

$$
d=\frac{n D}{2(n-1)} N A,
$$

где $d$ - диаметр источника выходного сигнала, $D-$ диаметр шариковой линзы, $n$ - показатель преломления, $N A$ - числовая апертура источника излучения.

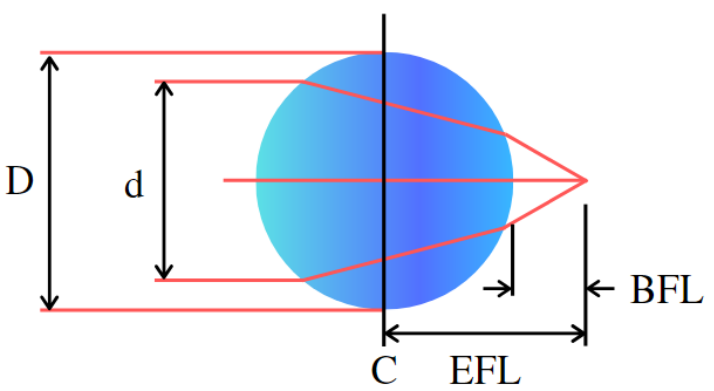

Рис. 4. Основные параметры шариковой линзы

Эти уравнения актуальны для точечных источников излучения или одномодового источника, но так как мы используем многомодовые оптические волокна, каждая мода пойдет по разным направлениям распространения. Данное уравнение будет использоваться для расчета расстояния BFL, которое в дальнейшем будем применять при практической реализации модели.

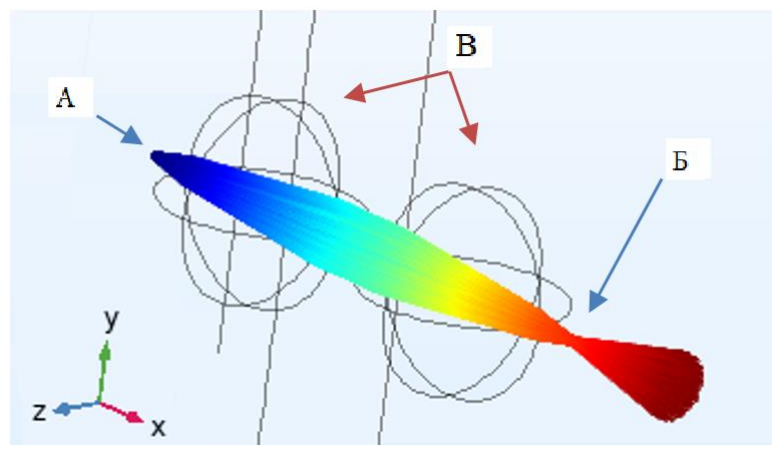

Рис. 5. Модель распространения излучения: $a$ - источник излучения, где должен будет располагаться тореи входного волокна, бперетяжка после системь линз, где будет располагаться тореи выходного волокна, в- иариковые линзы

После проведения расчетов была разработана модель геометрии распространения излучения (рис. 5) в программе COMSOL Multiphysics, с помощью которой определялась максимальная оптическая мощность передачи в сердцевину второго волокна, при изменении расстояния между торцом 
оптического волокна и поверхности линзы. Для расчета геометрии распространения излучения использовался матричный метод; для более точного построение модели шаг сетки задавался равным 0.1 мкм.

В табл. 1, 2 и 3 будут представлены результаты расчета для шариковых линз диаметрами 1, 2 и 3 мм соответственно. Для передачи излучения в ходе эксперимента были использованы многомодовые оптические волокна 105/125 кварц-кварц.

\section{1. Расчет шариковой линзы $\left(\mathrm{Al}_{2} \mathrm{O}_{3}\right)$ с диаметром 1 мм}

Эффективное фокусное расстояние шариковой линзы (1):

$$
E F L=\frac{n D}{4(n-1)}=\frac{1.756 \cdot 1}{4(1.756-1)}=0.581 \mathrm{MM} .
$$

Поскольку эта формула справедлива для точечного одномодового источника, то необходимо применить ее несколько раз для каждого из рассматриваемых положений торцов волокна. Таким образом, мы проанализируем оптическое пропускание от расстояния фокусного расстояния шарового объектива.

Для расчёта заднего фокусного расстояния шарового объектива $B F L$ (2) нужно знать значения $E F L$ и $D$ :

$$
B F L=E F L-\frac{D}{2}=0.581-0.5=0.081 \mathrm{Mм} .
$$

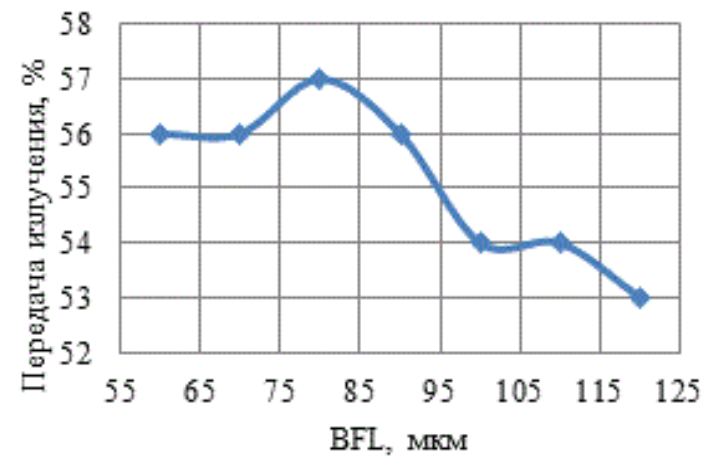

Рис. 6. Оптическое пропускание при моделировании эксперимента с диаметром линзы 1 мм

На рис. 6 представлены результаты, полученные из программной модели для сферической линзы диаметром 1 мм и $n=1.756$ :

$$
\begin{aligned}
& E F L=\frac{n D}{4(n-1)}=\frac{1.756 \cdot 2}{4 \cdot(1.756-1)}=1.161 \mathrm{MM}, \\
& B F L=E F L-\frac{D}{2}=1.161-1=0.161 \mathrm{MM} .
\end{aligned}
$$

Наибольший коэффициент передачи излучения составил $49 \%$.

\section{2. Расчет шариковой линзы $\left(\mathrm{Al}_{2} \mathrm{O}_{3}\right)$ с диаметром 2 мм}

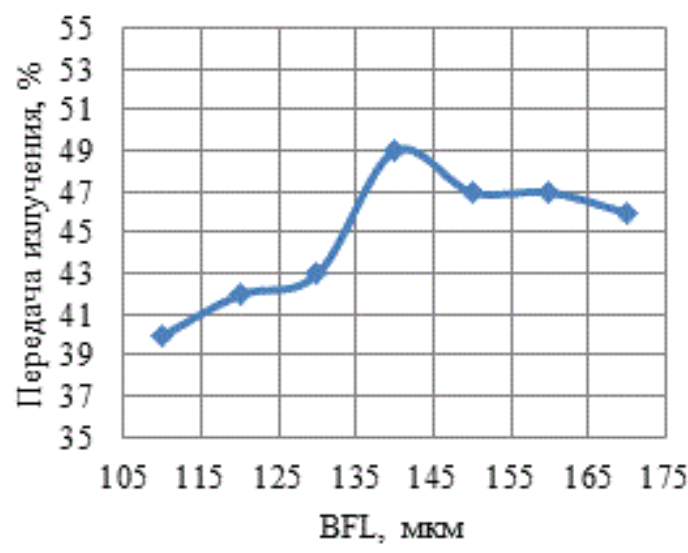

Рис. 7. Оптическое пропускание при проведении эксперимента с диаметров линзы 2 мм

На рис. 7 представлены результаты, полученные из программной модели для сферической линзы диаметром 2 мм и $n=1.756$ :

$$
\begin{aligned}
& E F L=\frac{n D}{4(n-1)}=\frac{1.508 \cdot 2}{4 \cdot(1.508-1)}=2.226 \mathrm{MM}, \\
& B F L=E F L-\frac{D}{2}=2.226-1.5=0.726 \mathrm{MM} .
\end{aligned}
$$

Наибольший коэффициент передачи излучения составил $41 \%$.

\section{3. Расчет шариковой линзы (K-8) с диаметром 3 мм}

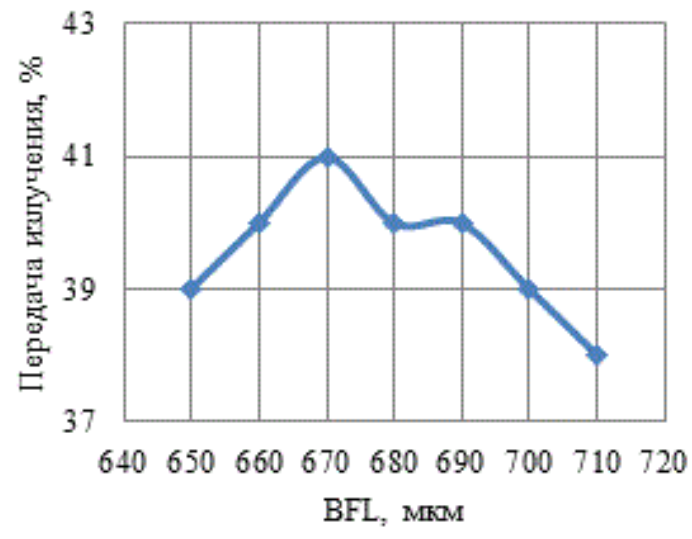

Рис. 8. Оптическое пропускание при проведении эксперимента с диаметров линзы 3 мм

На рис. 8 представлены результаты, полученные из программной модели для сферической линзы диаметром 3 мм и $n=1.508$ :

$$
E F L=\frac{n \cdot D}{4 \cdot(n-1)}=\frac{1.508 \cdot 3}{4 \cdot(1.508-1)}=2.226 \mathrm{MM},
$$




$$
B F L=E F L-\frac{D}{2}=2.226-1.5=0.726 \text { мм. }
$$

Наибольший коэффициент передачи излучения составил $41 \%$.

\section{3. Экспериментальная часть}

После теоретических расчетов был собран экспериментальный стенд, состоящий из:

1) лазерного диода накачки с длиной волны $975 \mathrm{HM}$;

2) оптического волокна Nufern MM-S105/ 125-22A;

3) шариковых линз диаметрами 1, 2 и 3 мм (для трех экспериментов);

4) измерителя оптической мощности Coherent FieldMax;

5) драйвера лазерного диода Gooch \& Housego;

6) микроподвижки ThorLabs NanoMax.

По схеме (рис. 3) были произведены замеры оптической мощности, прошедшей через систему линз.

После установки и закрепления микроподвижек первый отрезок волокна используется в качестве волокна доставки излучения: один его конец подключен к лазерному диоду, а второй закреплен в специальный держатель и установлен на микроподвижке. Второй отрезок волокна используется для анализа оптического пропускания: один его конец закреплен в держателе и установлен на микроподвижку, а второй соединен с измерителем оптической мощности.

В держатель, находящийся между торцами волокон, устанавливаются шариковые линзы с нужным диаметром. При этом шарики должны находиться друг от друга на расстоянии 1 мм.

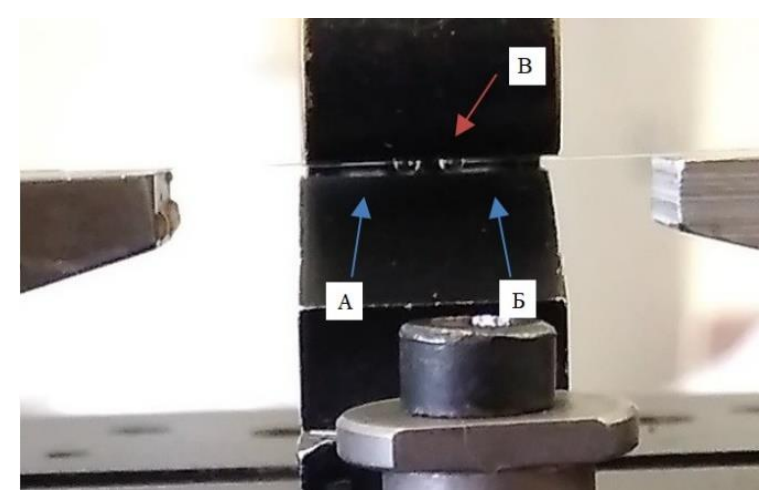

Рис. 9. Внешний вид экспериментального стенда (вид сбоку): $а$ - входное волокно, бвыходное волокно, с-линзы, закрепленные в держателе

В начале эксперимента торцы волокон должны быть приставлены к поверхности шариков. В дальнейшем торцы будут удаляться на расстояние $x$. Расстояние $x$ берется из теоретических расчетов, представленных в п. 2. На рис. 9 показан внешний вид экспериментального стенда.

Результаты трех экспериментов представлены в таблице.

Теоретические и экспериментальные результаты в трех экспериментах

\begin{tabular}{|l|l|c|}
\hline Диаметр и \\
показатель \\
преломления \\
$\begin{array}{l}\text { шариковой } \\
\text { линзы }\end{array}$ & $\begin{array}{l}\text { Полученная } \\
\text { оптиеская } \\
\text { мощность в } \\
\text { теории, \% }\end{array}$ & $\begin{array}{l}\text { Полученная } \\
\text { оптическая } \\
\text { мощность на } \\
\text { практике, \% }\end{array}$ \\
\hline 1 мм, $n=1.756$ & 57 & 62 \\
\hline 2 мм, $n=1.756$ & 49 & 51 \\
\hline 3 мм, $n=1.508$ & 41 & 39 \\
\hline
\end{tabular}

По данным из таблицы следует вывод, что погрешность теоретических расчетов и экспериментальных данных составили 5\%.

\section{4. Заключение}

В ходе проведенных работ был собран экспериментальный стенд c технологией Expanded Beam. Было проведено сравнение теоретических значений с экспериментальными данными. В результате значения практически совпали.

Коммерческие варианты рассматриваемой технологии обеспечивают коэффициент пропускания $87 \%$ для многомодовых оптических волокон. Разница между коммерческим и экспериментальным образцами составила $25 \%$, что можно объяснить отсутствием диэлектрического просветляющего покрытия, которое минимизирует френелевское отражение от поверхности оптического волокна и линз. Френелевское отражение составляет $4 \%$.

\section{Список литературы}

1. Летов Д. А., Токарева Я. Д. Исследование остаточной поляризации излучения эрбиевого суперлюминесцентного волоконного источника для волоконно-оптического гироскопа // Прикладная фотоника. 2018. Т. 5. № 4. С. 372-391.

2. Kazemi A. A. Fiber optic connectors for harsh environment of aviation and aerospace application // Proceeidings of SPIE. 2014. V. 9202. P. 1-15.

3. Tang Z., Zhang R., Mondal S. K., Shi F. G. Optimization of fiber-optic coupling and alignment tolerance for coupling between a laser diode and a wedged single-mode fiber // Optics Communications. 2001. V. 199. P. 95-101.

4. Saruwatari M., Sugie T. Efficient laser diode to single-mode fiber coupling using a combination of two lenses in confocal condition // IEEE Journal of Quantum Electronics. 1981. V. 17. P. 1021-1027

5. Cheung Y. M., Үіи C. H. Simulation of the alignment sensitivity on the coupling efficiency of a ball-lens capped TO-can laser diode source into a 
single-mode fiber // Proceedings of IEEE Conference on Electronic Materials and Packaging, 2002. P. 197-203.

6. Wagner R. E., Tomlinson W. J. Coupling efficiency of optics in single-mode fiber components. Applied Optics. 1982.V. 21. P. 2671-2688.

7. Niи J., Хи J. Coupling efficiency of laser beam to multimode fiber // Optics Communications. 2007. V. 274. P. 315-319.

8. Cook J. S., Mammel W. L., Grow R. J. Effect of misalignments on coupling efficiency of single-mode optical fiber butt joints // Bell System Technical Journal. 1973. V. 52. P. 1439-1448.

9. Chanclou P., Ramanitra H., Gravey P., Thual M. Design and performance of expanded mode fiber using microoptics // Journal of Lightwave Technology. 2002. V. 20. P. 836-842.

10. Вотинов Г. Н., Токарева Я. Д. Датчик высоких температур на основе волоконных брэгговских решеток // Прикладная фотоника. 2017. Т. 4. № 3. С. 222-229.

11. Гаранин А. И., Токарева Я. Д. Разработка стенда по исследованию параметров лазерного пучка на поверхности оптических объектов // Фотон-экспресс. 2019. № 3 (155). С. 16-20.

\section{References}

1. Letov D. A., Tokareva Ia. D. Research of residual polarization of radiation of an erbium superluminescent fiber source for a fiber-optical gyroscope. Applied Photonics, 2018, vol. 5, no. 4, pp. 372 391.

2. Kazemi A. A. Fiber optic connectors for harsh environment of aviation and aerospace application. Proceedings of SPIE, 2014, vol. 9202, pp. 1-15.
3. Tang Z., Zhang R., Mondal S. K., Shi F. G. Optimization of fiber-optic coupling and alignment tolerance for coupling between a laser diode and a wedged single-mode fiber. Optics Communications, 2001, vol. 199, pp. 95-101.

4. Saruwatari M., Sugie T. Efficient laser diode to single-mode fiber coupling using a combination of two lenses in confocal condition. IEEE Journal Quantum Electron, 1981, vol. 17, pp. 1021-1027.

5. Cheung Y. M., Yiu C. H. Simulation of the alignment sensitivity on the coupling efficiency of a ball-lens capped TO-can laser diode source into a single-mode fiber. Proceedings of IEEE Conference on Electronic Materials and Packaging, 2002, pp. 197-203.

6. Wagner R. E., Tomlinson W. J. Coupling efficiency of optics in single-mode fiber components. Applied Optics, 1982, vol. 21, pp. 2671-2688.

7. Niu J., Xu J., Coupling efficiency of laser beam to multimode fiber. Optics Communications, 2007, vol. 274, pp. 315-319.

8. Cook J. S., Mammel W. L., Grow R. J. Effect of misalignments on coupling efficiency of single-mode optical fiber butt joints. Bell System Technical Journal, 1973, vol. 52, pp. 1439-1448.

9. Chanclou P., Ramanitra H., Gravey P., Thual M. Design and performance of expanded mode fiber using microoptics. Journal of Lightwave Technology, 2002, vol. 20, pp. 836-842.

10. Votinov G. N., Tokareva Ya. D. High temperature sensor based on fiber bragg gratings. Applied photonics, 2017, vol. 4, no. 3, pp. 222-229.

11. Garanin A. I., Tokareva Ya. D. Design of a stand for measuring the diameter distribution of the mode field on the surface of optical objects. FotonExpress, 2019, no. 3 (155), pp. 16-20.

\section{Просьба ссылаться на эту статью в русскоязычных источниках следующим образом:}

Токарева Я. Д., Гаранин А. И., Петухова А. Ю., Конин Ю. А., Щербакова В. А. Разработка макета волоконно-оптического бесконтактного разъема с помощью технологии Expanded Beam // Вестник Пермского университета. Физика. 2020. № 1. С. 05-10. doi: 10.17072/1994-3598-2020-1-05-10

\section{Please cite this article in English as:}

Tokareva Ya. D., Garanin A. I., Petukhova A. Yu., Konin Yu. A., Scherbackova V. A. Layout design fiber optic proximity connector with Expanded Beam technology. Bulletin of Perm University. Physics, 2020, no. 1, pp. 0510. doi: 10.17072/1994-3598-2020-1-05-10 